\title{
L'agroécologie, une discipline aux confins de la science et du politique ${ }^{\star}$
}

\author{
Xavier Reboud ${ }^{1, *}$ et Étienne Hainzelin ${ }^{2}$ \\ ${ }^{1}$ Écologie, INRA, UMR Agroécologie, Dijon, France \\ 2 Agronomie, Conseiller du président, CIRAD, Ottawa, Canada
}

Pour saisir les ressorts de la montée de l'agroécologie dans ses différentes acceptions - scientifiques, politiques et sociales -, ce texte s'interroge sur la phase où une démarche scientifique se construit sur la base d'un questionnement sociétal et où se cherchent les articulations entre des préoccupations qui renvoient au politique et une argumentation scientifique destinée à la fois à leur donner consistance et légitimité et à les traduire en pistes d'action pour une diversité d'acteurs, des agriculteurs aux consommateurs. Dans ses grandes lignes, l'argumentation scientifique de l'agroécologie exploite l'idée d'un ressourcement de l'agronomie dans les principes de l'écologie pour mieux internaliser l'ensemble des conséquences d'envergure des choix agricoles. Cette quête de rupture avec l'agronomie «classique» interroge la capacité de l'interdisciplinarité à provoquer (puis justifier?) une évolution des perspectives à travers les concepts de sobriété, d'efficience, de résilience ou de robustesse. De plus, la nécessaire adaptation des systèmes agricoles et alimentaires aux conditions et aux besoins locaux conduit à souligner la dimension éminemment territoriale de l'agroécologie. Ceci induit la nécessité d'un élargissement de l'interdisciplinarité aux sciences de la société. Les difficultés du politique proviennent de cette recherche de rupture, des réorientations des politiques publiques qu'elle exige et des freins à lever.

\footnotetext{
‡ Voir dans ce numéro le texte d'introduction de Marcel Jollivet.

*Auteur correspondant : xavier.reboud@inra.fr
}

\section{Science et politique doivent favoriser le nécessaire changement pour rendre les systèmes agricoles et alimentaires plus durables}

La démographie, l'urbanisation, la libéralisation des marchés, la mondialisation des échanges, la persistance de l'insécurité alimentaire et nutritionnelle, la finitude de la planète Terre ou la croissance des préoccupations de santé publique dans une perspective One Health constituent autant de raisons de redéfinir, partout dans le monde, les enjeux de la production agricole et, plus généralement, des systèmes alimentaires. Après un siècle où la modernisation de l'agriculture était fondée sur la spécialisation, l'homogénéisation et l'artificialisation des fermes et des milieux, faisant la part belle dans les pays riches aux solutions chimiques associées à des variétés et des races essentiellement sélectionnées pour leurs performances productives, nombreux sont ceux qui aspirent à rappeler la nature profondément biologique et écologique de l'agriculture (Gliessman, 1990; Hainzelin, 2013). En réponse à un souci de durabilité, l'agriculture des pays du Nord est à la recherche de nouvelles voies d'intensification que celles qui ont présidé à son «industrialisation». Dans les pays du Sud, l'articulation de l'agriculture aux enjeux de la durabilité économique et sociale met avant tout en exergue la couverture des besoins alimentaires, la génération d'emplois et de revenus, de solidarité ou d'éducation; l'intensification de l'agriculture y est donc une obligation, mais selon des voies nouvelles. Au Nord comme au Sud, la transition vers une plus grande durabilité de l'agriculture bute toutefois sur la même faiblesse des médiations qui permettent de maintenir le cap face aux fragilités induites par les aléas climatiques, 
la volatilité des marchés ou encore l'expression d'intérêts contradictoires ou difficilement conciliables.

L'idée d'accroître la durabilité de l'agriculture a émergé dans les années $1970^{1}$ et n'a cessé de prendre de l'ampleur depuis. Le Millennium Ecosystem Assessment (2005) ou le rapport de l'IAASTD (Mc Intyre et al., 2009) ont contribué à souligner la nature profondément multifonctionnelle de l'agriculture. Par la suite, de nombreux travaux prospectifs ont exploré les conditions de la sécurité alimentaire mondiale d'une planète qui comptera plus de 9 milliards d'individus à l'horizon $2050^{2}$ : ces études illustrent la tension entre des objectifs antagonistes de productivité accrue et de couverture des services nécessaires pour assurer la durabilité des agroécosystèmes et des sociétés. Ils concluent à la nécessité d'arbitrages politiques alors même que les bases de connaissances scientifiques pour prendre les décisions sont incomplètes. L'agroécologie apparaît de façon croissante comme une voie majeure de progrès, mais il ne s'agira pas simplement de remplacer un modèle par un autre. Sa déclinaison dans différents contextes géographiques et socio-économiques se traduira dans une pluralité des chemins de la transition.

Dans ce contexte, les avancées scientifiques et la médiation politique représentent les deux voies essentielles d'accompagnement du changement, les premières en ouvrant le champ des possibles, la seconde en assurant la nécessaire régulation. La suite de ce texte en aborde quelques facettes qu'il explicite et en tire quelques conséquences.

\section{En remettant l'écosystème au centre, l'agroécologie se distingue de l'agronomie «classique»}

\section{Trois principes fondateurs de l'agroécologie cadrent les processus clé à respecter}

Si le débat occupe largement le champ politique et trouve sa concrétisation, au moins pour partie, dans le champ réglementaire, il n'est pour autant pas dénué de fondements scientifiques. À la base, c'est sans doute l'importance accordée à la durabilité environnementale qui conduit à aller chercher dans une science qui étudie les ressorts de fonctionnement de milieux naturels, les

\footnotetext{
${ }^{1}$ Silent spring, le livre de Rachel Carson (1962) est considéré comme un élément déclencheur des mouvements écologistes. ${ }^{2}$ Voir par exemple, pour un travail récent, les résultats de la prospective Agrimonde-Terra rendus publics en juin 2016 (http://institut.inra.fr/Missions/Eclairer-les-decisions/Prospec tives/Toutes-les-actualites/Prospective-Agrimonde-Terra).
}

règles qui pourraient s'appliquer aux agroécosystèmes pour l'atteindre. La remise en question des limites de l'agriculture « industrielle» par les sciences écologiques fonde les principes de base de l'agroécologie et renouvelle les approches en favorisant les regards interdisciplinaires. Les points de recouvrement entre les disciplines agronomie/écologie/agroécologie continuent de faire l'objet d'une interrogation au sein de la communauté scientifique elle-même ${ }^{3}$. Cette réflexion repose sur trois principes fondateurs. Le premier s'appuie sur l'idée d'une gestion plus sobre des ressources pour une moindre empreinte écologique de l'agriculture : il en découle la nécessité que les processus biologiques majeurs se substituent aux apports d'intrants (Magdoff, 2007). Cette substitution concerne aussi bien la gestion de la fertilité des sols et de l'eau que le contrôle de l'état sanitaire des cultures et des milieux. Agronomes et écologues s'entendent pour mettre en avant l'importance d'un premier principe de bouclage des cycles biogéochimiques impliquant notamment:

- l'augmentation de la qualité organique des sols et une minéralisation adaptée de la matière organique dans les horizons superficiels face à la demande nutritionnelle des plantes;

- l'optimisation des stocks, notamment de carbone et d'azote ainsi que des flux hydriques dans les sols. Le second principe énonce que des régulations naturelles sont susceptibles de se substituer au contrôle par des pesticides des principaux ravageurs.

Néanmoins, les lois de l'écologie dictent que l'on ne peut avoir d'auxiliaires des cultures sans proies pour les nourrir (Rosenzweig et Mac Arthur, 1963); la problématique, non close, de la gestion d'équilibres dynamiques entre populations en interactions souligne l'imbrication dans le temps et/ou l'espace entre zones de production et espaces semi-naturels qui alimente les débats entre le land sparing et le land sharing (Fischer et al., 2008). Le troisième principe, lié aux deux premiers (Duru et al., 2015), s'articule autour de l'importance accordée à la diversification. Cette dernière, mobilisable à différentes échelles spatiales, couvre une grande richesse d'effets bénéfiques. Elle se heurte toutefois à de nombreux freins, ceux qui précisément poussent à la simplification et spécialisation des exploitations et des territoires, là où les synergies entre activités variées ne sont pas suffisamment perceptibles par les acteurs, sont difficilement valorisables sur les seuls marchés privés ou en raison d'un retour sur investissement jugé de trop long terme.

\footnotetext{
${ }^{3}$ Voir par exemple dans l'ouvrage Protection agroécologique des cultures de Deguine et al. (2016), dans le chapitre 1 (Malézieux et al., 2016), le passage intitulé «L'agronomie estelle soluble dans l'agroécologie?».
} 


\section{La diversification, pilier incontournable de l'agroécologie}

La diversification est considérée comme un pilier incontournable de l'agroécologie pour plusieurs raisons (Reboud et Malézieux, 2015). La plus importante est sans doute qu'elle permet une meilleure occupation de l'espace ainsi que du sol au cours du temps avec des plantes n'exprimant pas toutes les mêmes besoins aux mêmes moments; elle accroît la productivité potentielle de biomasse par unité de surface. Cette propriété trouve sa traduction dans le calcul d'un ratio d'équivalent surface noté LER en anglais ${ }^{4}$ qui peut communément atteindre un gain de $40 \%$ par rapport aux cultures pures (Riley, 1984). Il s'agit là d'une exigence majeure de l'agroécologie, seule voie facilement mobilisable pour concilier le maintien global du volume de biomasse exportée par unité de surface et l'alimentation du fonctionnement biologique des agrosystèmes. Sans accroissement du LER, l'écologisation de l'agriculture ne peut qu'entraîner une baisse des rendements exportables, saufà les maintenir artificiellement par l'apport d'intrants.

La diversification accroît et facilite les flux d'énergie et de matière par une meilleure complémentarité des besoins entre compartiments donneurs et receveurs, concept repris dans la notion de métabolisme thermodynamique appliqué à l'échelle d'un agrosystème (Jorgensen et Svirezhev, 2004). À différentes échelles emboîtées, elle sert souvent de rempart naturel aux infestations qui trouvent plus difficilement les conditions qui leur sont favorables. Par nature, la diversification est aussi gage de résilience et robustesse accrues (Abson et al., 2013) face aux aléas et aux conditions inhabituelles (bet-hedging effect [Mattison et Norris, 2005]) à la fois par extension des adaptations présentes (des plantes supportant mieux les deux extrêmes de température, par exemple) et par propriété de redondance substitutive qui fait que si une espèce est affectée, une autre est là pour prendre le relais des fonctions non couvertes (Meynard et al., 2013).

Enfin, en plus de ses attributs d'ordre biologique qui s'expriment jusqu'à l'échelle des paysages, la diversification génère des besoins d'innovations techniques, économiques, organisationnelles qui se concrétisent à l'échelle des filières (Meynard et al., 2013) pour répondre aux besoins de collecte, stockage et transformation. Aussi, pour couvrir l'offre alimentaire locale, la diversification force à entretenir des capacités

\footnotetext{
${ }^{4}$ Le LER pour Land Equivalent Ratio est le ratio entre au numérateur la somme des rendements des cultures associées et, au dénominateur, le rendement des cultures pures correspondantes. Plus ce ratio est élevé, plus la productivité globale est proche du potentiel radiatif.
}

d'adaptation générique dans les territoires, dont les retombées s'exprimeront dans des agrosystèmes ayant une pérennité supérieure à celle de systèmes ultrasimplifiés rendus fragiles face aux aléas. En somme, en se donnant une contrainte apparente de court terme, la diversification génère une propriété dont le profit se réalise sur le moyen-long terme.

\section{La mise en œuvre des principes agroécologiques à l'épreuve des réalités}

\section{Des trois piliers de la durabilité, chacun s'approprie celui qui semble faire le plus défaut, ce qui renforce le caractère polysémique de l'agroécologie}

L'agroécologie comme mode de production durable cherche à combiner sobriété dans son recours aux intrants, respect des équilibres au sein de l'écosystème et résilience face aux aléas. Dans sa dimension politique ou militante, l'équité sera également visée. C'est la pluralité même de ces attentes d'ordre environnemental, économique ou social et leurs poids variables qui entretiennent le caractère polysémique de l'agroécologie: ceux pour qui la préoccupation environnementale domine les autres facettes de la durabilité y voient avant tout une façon de créer des écosystèmes écologiquement intensifs; pour d'autres, elle offre un modèle englobant des systèmes alimentaires; pour d'autres, il s'agit d'une discipline scientifique ou, plus pragmatiquement, d'un jeu de pratiques compatibles avec certains principes; cette gamme de sens va jusqu'à en faire un mouvement militant et politique en faveur de certaines formes d'agricultures, et même de sociétés (Wezel et al., 2009).

En France, l'ambition politique s'inscrit dans un plan «agroécologique ${ }^{5}$ que l'on peut qualifier d'ambitieux car il devance l'existence d'un jeu complet de références scientifiques et de pratiques, d'indicateurs, d'outils de pilotage et de suivi incontestables et facilement mobilisables. L'ampleur du décalage mérite d'être soulignée, car elle explique, au moins pour partie, les obstacles à la mise en œuvre dudit plan et à sa traduction en résultats tangibles.

\footnotetext{
${ }^{5}$ Le «projet agroécologique pour la France» élaboré et présenté dès décembre 2012 est inscrit dans la loi d'avenir pour l'agriculture, l'alimentation et la forêt, mise à jour le 15 mai 2017. Ce projet est traduit dans une série de programmes d'actions d'envergure nationale du ministère de l'Agriculture (programme national ambition bio 2017, plan Apiculture durable, etc.). Cette inscription dans la loi d'avenir conduit notamment à ce que l'agroécologie fasse l'objet d'une définition inscrite dans le code rural français.
} 


\section{La référence à l'idée de diversification varie selon les contextes}

Le déploiement de la diversification dans les territoires fait appel à des logiques différentes selon ce qui fait le plus défaut. Pour l'agriculture industrielle, la diversification à des échelles fines peut être un moyen de gérer des ravageurs et de mieux exploiter les ressources (par exemple, via l'agroforesterie, l'enherbement de la vigne...). À des échelles intermédiaires, la diversification sera davantage perçue comme un moyen de réduire la dépendance aux intrants (par exemple, quand la richesse de l'assolement prévient le risque phytosanitaire (Schott et al., 2010; Meynard et al., 2013). Elle peut de plus induire la valorisation des produits secondaires, surtout en situation de polyculture - élevage (Hansen, 1996; Lin, 2011). Dans les pays du Sud, ce même thème de diversification couvre des réalités et des pratiques différentes : agroforesterie pour maximiser la production de biomasse tout en réduisant les risques, stratégie "push-pull» pour repousser les ravageurs vers la bordure des parcelles de production, méthodes de semis direct sous couvert végétal pour gérer l'eau et l'érosion, diversification des productions pour couvrir les besoins alimentaires et nutritionnels locaux. Tout autant que re-diversifier, il s'agit surtout de mieux piloter la diversité existante. Quel que soit l'endroit, le choix d'une stratégie de diversification peut représenter une réelle contrainte de mobilisation des savoirs et compétences éparses ou de complexification des débouchés. En outre, les avantages de la diversification s'expriment souvent de manière différée dans le temps ou seront décalés dans l'espace. Une illustration de ce point concerne l'assolement: tout agriculteur qui introduit une culture inédite contribue à diminuer les charges parasitaires des cultures de ses voisins sans en toucher un quelconque retour. De ce point de vue, la diversification souffre des limites que l'on attribue à la fourniture de biens communs (Feeny et al., 1990).

\section{Des concepts en agronomie et en écologie à l'identification de besoins de gestion des territoires et d'organisation des marchés}

Une conséquence directe des principes de l'agroécologie est de tirer au mieux profit du contexte local et de la biodiversité qui y est déjà présente. Par la diversification, au moyen d'espèces et de variétés localement adaptées, l'agroécologie vise explicitement à maximiser la production de biomasse dans le temps et l'efficience de l'usage des ressources. Elle se traduit toutefois par un besoin de caractérisation du contexte environnemental bien supérieur à celui qui est traditionnellement requis. Dans certaines zones d'agriculture intensive, cette exigence conduit à mieux caractériser les hétérogénéités du contexte à l'aide de capteurs et de cartographies, pour ajuster ensuite des apports différenciés et ainsi accroître l'efficience des intrants. Elle suppose aussi de mieux s'appuyer sur les savoirs locaux des producteurs, car l'efficacité des leviers proposés peut s'avérer très dépendante du contexte de leur mise en pratique. On s'éloigne donc d'une agronomie générique, i.e., d'application à tous les contextes, dans la mesure où une large partie des innovations repose sur une connaissance fine des conditions locales, que ce support se fasse par de nouvelles approches de caractérisation $\mathrm{du}$ milieu ou repose sur la valorisation du savoir-faire des agriculteurs. Pour couvrir les différentes facettes de la durabilité, l'agroécologie apparaît ainsi comme soutenant l'ambition très moderne de valorisation et de partage des ressources, en réussissant le mariage de la science et des connaissances traditionnelles pour réunir la compréhension profonde de la complexité à la sobriété et la simplicité des interventions de sa gestion, ce qui signifie donner une plus grande place aux systèmes locaux d'innovation.

Le consommateur a relativement peu de prise directe sur la dimension environnementale de ce qu'il consomme alors qu'il semble plus facilement acteur de ses dimensions sociales (circuits courts, achats équitables). S'il n'y a pas de reconnaissance de l'effort environnemental par le consommateur (via le prix) ou par le contribuable (via des aides), il est difficile de contrer la spécialisation et la simplification dans des économies d'échelle mondialisées en concurrence et où dominent souvent les seuls intérêts marchands privés. À n'en pas douter, un moyen de relier consommation et préservation de l'environnement (local) consiste à miser sur la proximité entre producteur et consommateur pour renforcer la visibilité et le partage d'intérêts communs. Tenir compte des attributs sociaux des produits (produits équitables, agriculture familiale ou dense en emplois) fait partie de la même réflexion sur la durabilité que la référence aux enjeux environnementaux globaux (changement climatique et biodiversité). Au Nord, les circuits courts restent marginaux, malgré leur forte croissance, alors qu'ils dominent dans de nombreux pays du Sud; aussi les dynamiques de proximité entre producteurs et consommateurs, s'exprimeront-elles de manière différentiée. Les tentatives pour relier proximité et durabilité, qui ménagent un rôle de médiation aux consommateurs, se retrouvent dans :

- les appellations d'origine contrôlée, ou plus largement les indications géographiques, constituant une alternative à cette homogénéisation déspatialisée mais ne concernant généralement pas les produits de première nécessité ;

- la reconnaissance via les labels octroyés du respect d'un cahier des charges technique ou éthique, sans dimension géographique explicite; 
- enfin la promotion des circuits directs et de proximité qui suppriment les échelons intermédiaires et qui permettent à la fois de maintenir la diversification de gamme et de promouvoir conjointement différents critères qui, sinon, resteraient dissociés (concept de panier/bouquet de biens et services).

Pour autant, ces initiatives peinent à se généraliser à une large échelle. En poussant plus loin cette logique, on pourrait imaginer que la diversification puisse s'appuyer sur le concept de «territoire intelligent», associant les regards sur les filières et sur les dynamiques territoriales où les achats de denrées agricoles répondraient non seulement à la couverture des besoins nutritionnels, mais pourraient aussi venir en support d'actions de développement, de formation ou d'expression d'une solidarité pouvant orienter vers les systèmes les plus résilients (Lescourret et al., 2015). Mais ces politiques en faveur du développement local butent sur d'autres aspects globaux de couverture des besoins de populations en forte croissance. Une absence de gouvernance mondiale des échanges comme d'une entente minimum sur les règles à respecter pour assurer la durabilité face à des enjeux planétaires rend difficile l'émergence de solutions.

\section{Retour sur le politique : entre volontarisme et pragmatisme}

Une certaine mobilisation politique tend à s'articuler autour de l'objectif d'encourager les modes de production multi-performants via la promotion de l'agroécologie. Cela se fait toutefois dans un contexte dont le fond est constitué par des politiques globalement en faveur de la modernisation de l'agriculture au sens large, de la PAC à la révolution verte, où les intérêts marchands, privés et immédiats dominent encore, au détriment d'autres logiques non marchandes, alliant privé et public, court et plus long terme. Devant le nombre de situations possibles de blocage ou d'intérêts contrariés, les politiques tentent de s'appuyer sur la cohérence d'ensemble de toutes les dimensions de l'exploitation, des filières et des territoires. Mais si les politiques poussent à mieux articuler les échelles, il reste douteux que tous ces éléments puissent se déployer aisément dans un marché mondialisé. Actuellement, il reste encore difficile de cerner le ou les éléments moteurs qui permettront de basculer dans un nouveau paradigme.

Dans la loi d'avenir pour l'agriculture, l'alimentation et la forêt, la France a visé la mise en œuvre concrète de l'agroécologie pour améliorer les performances économiques, environnementales et sociales des exploitations agricoles. La valence environnementale est clairement mise en avant dans les actions entreprises dans différents secteurs comme l'agroforesterie, l'apiculture durable, etc. (Fig. 1), la sobriété du recours aux pesticides et aux antibiotiques étant cadrée par les plans Ecophyto ${ }^{6}$ et Antibio $^{7}$, respectivement. Les retombées sur les services écosystémiques pourront bénéficier des avancées réalisées dans le cadre de l'évaluation EFESE ${ }^{8}$. Début 2016, l'État français annonçait avoir déjà mis en œuvre $81 \%$ de sa loi, à travers 73 mesures réglementaires d'application ${ }^{9}$. Toutefois, seules 14 mesures ciblent explicitement et conjointement les performances économiques et environnementales. Certaines mesures misent surtout sur «la force du collectif et les démarches ascendantes des territoires ${ }^{10}$ ", notamment en labellisant des Groupements d'intérêt économique et environnemental (GIEE). Ces mesures de labellisation visent assez directement à apporter une reconnaissance aux efforts consentis pour élargir les critères de conduite et d'évaluation de la performance des exploitations selon les différents piliers de la durabilité. Cette reconnaissance est une étape importante pour enclencher une transition vers un nouveau modèle de production. On

\footnotetext{
${ }^{6}$ Le plan Ecophyto, maintenant dans sa seconde version, coordonne une série de mesures pour couvrir l'objectif de réduire progressivement l'utilisation des produits phytopharmaceutiques en France de $25 \%$ à l'horizon 2020 puis $50 \%$ à l'horizon 2025. Un plan d'action est transcrit dans la loi du 6 février 2014, dite "loi Labbé», qui interdit la vente aux particuliers de produits phytopharmaceutiques, et contraint l'utilisation de ces produits dans les espaces verts, forêts, et promenades gérés par des personnes publiques. Une autre partie concerne la mise en place du dispositif des «Certificats d'économies de produits phytopharmaceutiques ».

${ }^{7}$ Politique publique pilotée par le ministère de l'Agriculture, le plan Ecoantibio vise la réduction des risques d'antibiorésistance en médecine vétérinaire. Il s'inscrit pleinement dans les recommandations pour préserver l'efficacité des antibiotiques édictées par de grands organismes internationaux (OIE, OMS, FAO et instances européennes). Il repose largement sur un moindre recours aux antibiotiques et un cadrage réglementaire interdisant certains usages tel que l'utilisation en additif dans l'alimentation des animaux.

${ }^{8}$ L'ampleur de l'érosion de la biodiversité a conduit les scientifiques à qualifier la période actuelle de $6^{\mathrm{e}}$ grande extinction. Face à ce constat, une mobilisation généralisée a vu le jour pour qualifier et quantifier les implications sur le fonctionnement de la planète à travers la couverture de grands services écosystémiques. L'analyse est conduite à l'échelle des pays. Sa déclinaison pour la France est connue sous le nom d'EFESE pour «Évaluation française des écosystèmes et des services écosystémiques ». (voir http://www.developpementdurable.gouv.fr/levaluation-francaise-des-ecosystemes-et-desservices-ecosystemiques).

${ }^{9} \mathrm{http}$ ://agriculture.gouv.fr/la-loi-davenir-en-chiffres.

${ }^{10} \mathrm{http}$ ://agriculture.gouv.fr/titre-i-performance-economiqueet-environnementale-des-filieres-agricoles-et-alimentaires.
} 


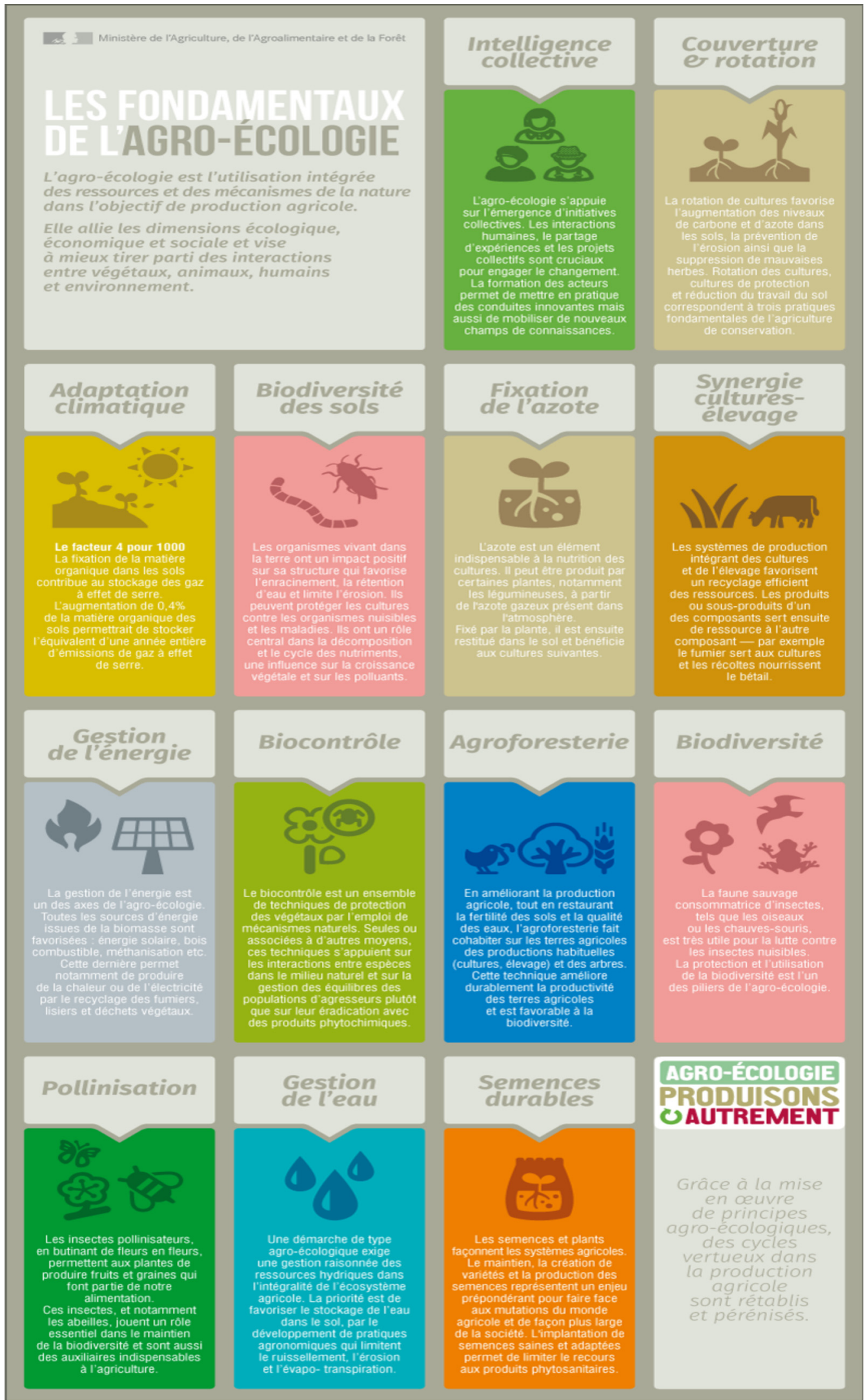

Fig. 1. Les fondamentaux de l'agroécologie (source: Ministère de l'Agriculture et de l'Alimentation, http://infographies. agriculture.gouv.fr/-Licence CC-BY-NC-ND 4.0 $)^{11}$. 
doute toutefois qu'elle puisse y parvenir seule, car ce serait admettre que les modes de consommation n'ont pas besoin de se transformer parallèlement à ceux de la production, et que le système intensif existant au Nord contiendrait déjà la majorité des éléments de son évolution - et pourrait donc être ajusté à la marge.

Par sa loi d'orientation, la France fait un peu figure de pionnier au plan mondial, alors que la majorité des pays du monde restent sur une vision conventionnelle de l'intensification de l'agriculture: l'agroécologie y est souvent cantonnée à une simple revendication sociale ou technique. Sur le plan international, la FAO a organisé dès septembre 2014, une série de conférences mondiales «multi-stakeholders» sur l'agroécologie. Certains pays ont ainsi pu s'engager dans un rôle de catalyseur dans la transformation des agendas politiques et techniques des agricultures du monde. Dans le contexte de l'année internationale des agricultures familiales, un «Club des pays amis de l'agroécologie» a pu afficher son engagement volontariste notamment pour faire reconnaître les préconisations agroécologiques comme une base à la fois légitime (notamment $\mathrm{du}$ fait de ses justifications scientifiques) et partageable pour faire évoluer les agricultures du monde.

Ainsi, une tension existe entre le plaidoyer pour la rupture agroécologique, poussé parfois jusqu'à la caricature, et le pragmatisme qui consiste à promouvoir divers chemins de transition. L'agroécologie, qui oblige à renouveler la façon de mesurer la performance ou la valeur de la production, et à renforcer la dimension locale, bouleverse les missions d'une action publique souvent organisée à une échelle plutôt nationale. Les principes qu'elle prône, en conduisant à donner du poids aux bénéfices de moyen terme et sur des biens communs au détriment de simplifications et d'optimisation de court terme, constituent pour cette même action publique un autre défi. Une difficulté majeure consiste précisément à trouver, dans les nouvelles approches scientifiques et techniques que l'interdisciplinarité peut générer, la batterie de critères justifiant les actions publiques à même de provoquer le changement. C'est en se donnant les moyens de rendre compte de la valeur du non

\footnotetext{
${ }^{11}$ Affiche publiée par le ministère français en charge de l'agriculture en 2015 qui résume la déclinaison de ce que recouvre sa vision de l'agroécologie en miroir des grandes lignes de déploiement d'un plan d'action. On notera que certaines actions relèvent peu d'une déclinaison de principes de l'agroécologie au sens strict, mais visent surtout à susciter une mobilisation généralisée auprès d'une gamme élargie d'acteurs à des échelles d'actions balayant du local au supranational. Consulter cette affiche à l'adresse source http:// agriculture.gouv.fr/infographie-les-fondamentaux-de-lagroecologie.
}

marchand et des bilans de long terme et de grande ampleur, que l'agroécologie pourra à la fois accomplir son ambition et se développer.

\section{Remerciements}

Ce texte a bénéficié des échanges scientifiques au sein du groupe «Agroécologie et sol» de l'alliance pour l'environnement, AllEnvi. Le rapporteur ainsi que différents contributeurs F. Maraux, F. Lescourret et H. Guyomard ont largement contribué à son amélioration.

\section{Références}

Abson D.J., Fraser E.D., Benton T.G., 2013. Landscape diversity and the resilience of agricultural returns: a portfolio analysis of land-use patterns and economic returns from lowland agriculture, Agriculture \& food security, 2, 1, 1 .

Carson R., 1962. Silent spring, Boston, Houghton Mifflin.

Deguine J.-P., Gloarec C., Laurent P., Ratnadass A., Aubertot J.-N. (Eds.), 2016. Protection agroécologique des cultures, Versailles, éditions Quae.

Duru M., Therond O., Martin G., Martin-Clouaire R., Magne M.-A., Justes E., Journet E.-P., Aubertot J.-N., Savary S., Bergez J.-E., Sarthou J.-P., 2015. How to implement biodiversity-based agriculture to enhance ecosystem services: a review, Agronomy for Sustainable Development, 35, 4, 1259-1281.

Feeny D., Berkes F., McCay B.J., Acheson J.-M., 1990. The tragedy of the commons: twenty-two years later, Human Ecology, 18, 1, 1-19.

Fischer J., Brosi B., Daily G.C., Ehrlich P.R., Goldman R., Goldstein J., Lindenmayer D.B., Manning A.D., Mooney H.A., Pejchar L., Ranganathan J., Tallis H., 2008. Should agricultural policies encourage land sparing or wildlife friendly farming? Frontiers in Ecology and the Environment, 6, 7, 380-385.

Gliessman S.R., 1990. Agroecology: researching the ecological basis for sustainable agriculture, in Agroecology: researching the ecological basis for sustainable agriculture, New-York, Springer, 3-10.

Hainzelin E., 2013. Introduction, in Hainzelin E. (Ed.), Cultivating Biodiversity to Transform Agriculture, The Netherlands, Springer, 261, 1-10.

Hansen J.W., 1996. Is agricultural sustainability a useful concept? Agricultural Systems, 50, 2, 117-143.

Jorgensen S.E., Svirezhev Y.M., 2004. Towards a thermodynamic theory for ecological systems, Elsevier, Amsterdam, $368 \mathrm{p}$.

Lescourret F., Magda D., Richard G., Adam-Blondon A.F., Bardy M., Baudry J., Doussan I., Dumont B., Lefèvre F., Litrico I., Martin-Clouaire R., Montuelle B., Pellerin S., Plantegenest M., Tancoigne E., Thomas A., Guyomard H., Soussana J.-F., 2015. A social-ecological approach to managing multiple agro-ecosystem services, Current Opinion in Environmental Sustainability, 14, 68-75. 
Lin B.B., 2011. Resilience in agriculture through crop diversification: adaptive management for environmental change, BioScience, 61, 3, 183-193, https://academic.oup. com/bioscience/article/61/3/183/238071/Resilience-inAgriculture-through-Crop.

Magdoff F., 2007. Ecological agriculture: Principles, practices, and constraints, Renewable Agriculture and Food Systems, 22, 2, 109-117.

Malézieux E., Jarne P., Aubertot J.-N., Boiffin J., Lescourret F., Lauri P.-E., 2016. Les contours de l'agroécologie, in Deguine J.-P., Gloarec C., Laurent P., Ratnadass A., Aubertot J.-N. (Eds.), Protection agroécologique des cultures, Versailles, éditions Quae, 23-40.

Mattison E.H., Norris K., 2005. Bridging the gaps between agricultural policy, land-use and biodiversity, Trends in Ecology \& Evolution, 20, 11, 610-616, http://www.science direct.com/science/article/pii/ S0169534705002685.

Mc Intyre B.D., Herren H.R., Wakhungu J., Watson R.T. (Eds.), 2009. Agriculture at a crossroads. Global report, International assessment of agricultural knowledge, science and technology for development (IAASTD), Washington, Island Press.

Meynard J.-M., Messéan A., Charlier A., Charrier F., Le Bail M., Magrini M.-B., Savini I., 2013. Freins et leviers à la diversification des cultures: étude au niveau des exploitations agricoles et des filières, Oléagineux, Corps gras,
Lipides, 20, 4, D403, https:/www.ocl-journal.org/articles/ ocl/pdf/2013/04/ocl20-4- D3. pdf.

Millennium Ecosystem Assessment (MEA), 2005. Ecosystems and Human Well-being: Synthesis, Washington D.C., Island Press, http://www.millenniumassessment.org/documents/ document.356.aspx.pdf.

Reboud X., Malézieux E., 2015. Vers une agroécologie biodiverse: enjeux et principaux concepts mobilisés, Innovations Agronomiques, 43, 1-6, http://www6.inra.fr/ ciag/content/download/5592/42504/file/Vol43-1-Reboud. pdf.

Riley J., 1984. A general form of the 'land equivalent ratio', Experimental agriculture, 20, 1, 19-29.

Rosenzweig M.L., Mac Arthur R.H., 1963. Graphical representation and stability conditions of predator-prey interactions, The American Naturalist, 97, 895, 209-223.

Schott C., Mignolet C., Meynard J.M., 2010. Les oléoprotéagineux dans les systèmes de culture: évolution des assolements et des successions culturales depuis les années 1970 dans le bassin de la Seine, Oléagineux, Corps gras, Lipides, 17, 5, 276-291, https:/www.ocl-journal.org/en/ articles/ocl/pdf/2010/05/ocl2010175p276.pdf.

Wezel A., Bellon S., Doré T., Francis C., Vallod D., David C. 2009. Agroecology as a science, a movement and a practice. A review, Agronomy for Sustainable Development, 29, 4, 503-515.

Citation de l'article : Reboud X., Hainzelin É., 2017. L'agroécologie, une discipline aux confins de la science et du politique. Nat. Sci. Soc. 25, S64-S71. 